\title{
Article
}

\section{Postpartum Gray Matter Changes in the Auditory Cortex}

\author{
Eileen Luders $1,2,3, *$, Christian Gaser ${ }^{4}$, Malin Gingnell ${ }^{2,5}$, Jonas Engman ${ }^{5}$, Inger Sundström Poromaa ${ }^{2}$ \\ and Florian Kurth ${ }^{1}$
}

check for updates

Citation: Luders, E.; Gaser, C.;

Gingnell, M.; Engman, J.; Sundström Poromaa, I.; Kurth, F. Postpartum Gray Matter Changes in the Auditory Cortex. J. Clin. Med. 2021, 10, 5616. https://doi.org/10.3390/jcm10235616

Academic Editor: Moussa Antoine Chalah

Received: 22 October 2021

Accepted: 27 November 2021

Published: 29 November 2021

Publisher's Note: MDPI stays neutral with regard to jurisdictional claims in published maps and institutional affiliations.

Copyright: (C) 2021 by the authors Licensee MDPI, Basel, Switzerland. This article is an open access article distributed under the terms and conditions of the Creative Commons Attribution (CC BY) license (https:/ / creativecommons.org/licenses/by/ $4.0 /)$.
1 School of Psychology, University of Auckland, Auckland 1010, New Zealand; f.kurth@auckland.ac.nz

2 Department of Women's and Children's Health, Uppsala University, 75105 Uppsala, Sweden; malin.gingnell@akademiska.se (M.G.); inger.sundstrom@kbh.uu.se (I.S.P.)

3 Laboratory of Neuro Imaging, School of Medicine, University of Southern California, Los Angeles, CA 90089, USA

4 Departments of Psychiatry and Neurology, Jena University Hospital, 07747 Jena, Germany; christian.gaser@uni-jena.de

5 Department of Psychology, Uppsala University, 75105 Uppsala, Sweden; jonas.engman@akademiska.se

* Correspondence: e.lueders@auckland.ac.nz

\begin{abstract}
After giving birth, a mother's brain undergoes functional adaptations fostering the ability to properly respond to the needs of her newborn. Tuning into and understanding her baby's crying is among the top skills required and executed in the early stages of motherhood. However, surprisingly little is known about potential changes in the anatomy of the maternal auditory cortex. Therefore, in this longitudinal study, we compared the brains of 14 healthy women between immediate postpartum (within 1-2 days of childbirth) and late postpartum (at 4-6 weeks after childbirth), focusing on areas of the primary, secondary, and higher auditory cortex. We observed significant volume increases within all auditory regions and subregions examined, which might reflect rapid adaptations of the mother's brain in relation to reliably interpreting her newborn's cries. There was also a trend for a larger postpartum increase within right-hemispheric regions compared to left-hemispheric regions that might be specifically linked to the ability to discern the pitch, sound, and volume of a baby's crying. Follow-up research is warranted to replicate these findings and evaluate their current interpretation.
\end{abstract}

Keywords: auditory cortex; brain plasticity; gray matter; MRI; postpartum; pregnancy

\section{Introduction}

Mothers' brains show links to their baby's cries on a functional level. Scientific evidence for this natural phenomenon has been provided, for example, in neurophysiological experiments measuring brain activity in response to infant crying using functional magnetic resonance imaging or magnetoencephalography [1-4]. These studies have not only demonstrated that there is indeed an early crying-specialized activity in a mother's brain, but also that the auditory cortex is among those regions that showed such an activity. Animal studies have provided insights into possible underlying mechanisms by pointing to the effect of hormones in the framework of infant-mother attachment [5]. Oxytocin in the auditory cortex of female mice, for example, increased the salience of acoustic social stimuli and, as such, enabled and enhanced pup retrieval behavior [6].

The question arises of whether the aforementioned functional, hormonal, and behavioral phenomena are accompanied by actual macroscopic changes within the auditory cortex. While at least two prior postpartum studies revealed gray matter increases throughout the brain that also encompassed the auditory cortex $[7,8]$, structural imaging studies specifically targeting the maternal auditory cortex after giving birth are missing. Therefore, here we applied an advanced region-of-interest technique to gain further insights into possible physical changes of auditory brain regions when transitioning to motherhood. More specifically, we examined postpartum changes in brain tissue for three auditory main 
regions and five subregions, specifically Te1 consisting of Te1.0, Te1.1, and Te1.2 [9]; Te2 consisting of Te2.1 and Te2.2 [10]; and Te3 [11]. For the exact location and definition of these areas, please refer to [9-11]. Te1 constitutes the primary auditory cortex (or Brodmann Area 41); Te2 may be considered the secondary auditory cortex (or Brodmann Area 42); and Te3 constitutes part of the higher auditory cortex (corresponding to the posterior 2/3 of Brodmann Area 22).

Aside from establishing which auditory (sub)regions will show changes-presumably increases rather than decreases, given the prior findings [7,8]—our study will reveal if there are different effects pertaining to left and right auditory regions. Such laterality effects are not unlikely. For example, prior analyses in mice have demonstrated that pup retrieval depends on the functional activity of the left ear, but not the right ear [6]. The same study also reported that retrieval behavior was accelerated by oxytocin and that oxytocin receptors were preferentially expressed in the left auditory cortex. Thus, any structural changes within auditory regions in the current study might be more pronounced in the left hemisphere than the right hemisphere. On the other hand, left Brodmann Area 22-as part of Wernicke's area-has been implicated in language comprehension and speech production [12], whereas right Brodmann Area 22 is involved in discriminating pitch, sound, and volume. Thus, it seems equally possible that changes occurring during the first few weeks after giving birth are more pronounced in the right hemisphere than the left hemisphere, at least with respect to Te3.

\section{Materials and Methods}

Our sample included 14 healthy postpartum women, aged between 25 and 38 years (mean \pm SD: $32.8 \pm 4.0$ years). All women were Caucasian, and 13 women $(92.9 \%$ ) were of Nordic origin. 13 women $(92.9 \%)$ were married or cohabiting, and 11 women $(78.6 \%)$ had an education on the university level. All women were recruited from the maternity ward at Uppsala University Hospital (Sweden) and had an uncomplicated delivery ( $n=9$ vaginal $/ n=5$ Caesarean); for 7 women $(50 \%)$ it was a first-time delivery. For study exclusion criteria, please see [13]. All women underwent brain scanning at two time points, 1-2 days after delivery (immediate postpartum) and 4-6 weeks after delivery (late postpartum). All women provided written informed consent and all procedures were approved by the Regional Ethical Review Board, Uppsala (Sweden). Readers may seek access to the data through the corresponding author.

Brain images were acquired on a Philips Achieva 3T-X scanner using a phase-sensitive inversion recovery T1-weighted sequence and the following parameters: $5700 \mathrm{~ms}$ repetition time, $15 \mathrm{~ms}$ echo time, $400 \mathrm{~ms}$ inversion time, 90 degrees flip angle, $23 \mathrm{~cm}$ field of view, and $0.45 \times 0.45 \times 2.0 \mathrm{~mm}^{3}$ voxel size. Data processing was done in Matlab (http:// www.mathworks.com/products/matlab, accessed on 22 October 2021) using the VBM8 toolbox (http:/ / dbm.neuro.uni-jena.de/vbm, accessed on 22 October 2021) and a workflow optimized for longitudinal processing, as detailed elsewhere [13]. The procedure resulted in one image for each woman reflecting the difference in voxel-wise gray matter between immediate and late postpartum, with positive values indicating an increase and negative values a decrease. These difference images were multiplied with the cytoarchitectonic tissue probabilities for the regions of interest in each hemisphere (Te1.0, Te1.1, Te1.2, Te2.1, $\mathrm{Te} 2.2$, and $\mathrm{Te} 3$ ), as detailed elsewhere [14]. The required cytoarchitectonic probability maps are based on post mortem data [9-11,15] and freely available via EBRAINS (https: / / ebrains.eu/, accessed on 22 October 2021).

The region-specific volumetric changes were calculated in cubic millimeters $\left(\mathrm{mm}^{3}\right)$ by adding the voxel-wise probability-weighted gray matter change across all voxels pertaining to each region of interest (Te1.0, Te1.1, Te1.2, Te2.1, Te2.2, and Te3). In addition, the total volume for the primary auditory cortex (Te1) was calculated as the sum of Te1.0, Te1.1, and Te1.2; the total volume for the secondary auditory cortex (Te2) was calculated as the sum of Te2.1 and Te2.2. One-sample t-tests were applied to detect possible volume changes after confirming that assumptions for parametric testing were met. Significance levels were 
adjusted for multiple comparisons by controlling the false discovery rate (FDR) [16,17]. Moreover, we calculated the regional changes in percent $(\%)$ and tested whether the changes in one hemisphere were larger than in the other. For this purpose, we applied paired t-tests using FDR-corrections, again after ensuring that assumptions for parametric testing were met.

\section{Results}

There were no significant decreases between immediate postpartum (within 1-2 days of childbirth) and late postpartum (at 4-6 weeks after childbirth) for Te1, Te2, T3, or any of the subregions. In contrast, there were significant increases between these two time points for all regions of interest. Cohen's $d$ as well as statistical T- and FDR-corrected p-values (q values) are provided in Table 1; the region-specific changes (in \%) are visualized in Figure 1. With respect to possible hemispheric differences, there was a trend for a significantly larger increase of right TE2.1 and right TE3 compared to left TE2.1 and left TE3 (both $\mathrm{q} \leq 0.052$ ).

Table 1. Changes in region-specific volumes between immediate and late postpartum.

\begin{tabular}{|c|c|c|c|c|c|c|}
\hline \multirow{2}{*}{ Region } & \multicolumn{3}{|c|}{ Left Hemisphere } & \multicolumn{3}{|c|}{ Right Hemisphere } \\
\hline & Cohen's d & $\mathrm{T}$ & $q^{1}$ & Cohen's d & $\mathbf{T}$ & $q^{1}$ \\
\hline $\mathrm{Te} 1$ & 2.9346 & 5.2904 & 0.0002 * & 2.5234 & 4.5491 & 0.0004 * \\
\hline $\mathrm{Te} 1.1$ & 2.5349 & 4.5699 & 0.0004 * & 2.9113 & 5.2485 & 0.0002 * \\
\hline Te1.0 & 1.214 & 2.1885 & 0.0237 * & 1.4394 & 2.595 & 0.0118 * \\
\hline $\mathrm{Te} 1.2$ & 2.6764 & 4.8249 & 0.0003 * & 2.7033 & 4.8734 & 0.0003 * \\
\hline $\mathrm{Te} 2$ & 3.6535 & 6.5864 & 0.0001 * & 2.5808 & 4.6527 & 0.0004 * \\
\hline $\mathrm{Te} 2.1$ & 3.1287 & 5.6404 & 0.0002 * & 3.9615 & 7.1416 & 0.0001 * \\
\hline TE2.2 & 3.5196 & 6.3451 & $0.0001 *$ & 2.1455 & 3.8679 & 0.0012 * \\
\hline $\mathrm{Te} 3$ & 2.078 & 3.7462 & 0.0014 * & 2.7147 & 4.894 & 0.0003 * \\
\hline
\end{tabular}

${ }^{1}$ q-values are FDR-corrected $p$-values; * denotes significance.
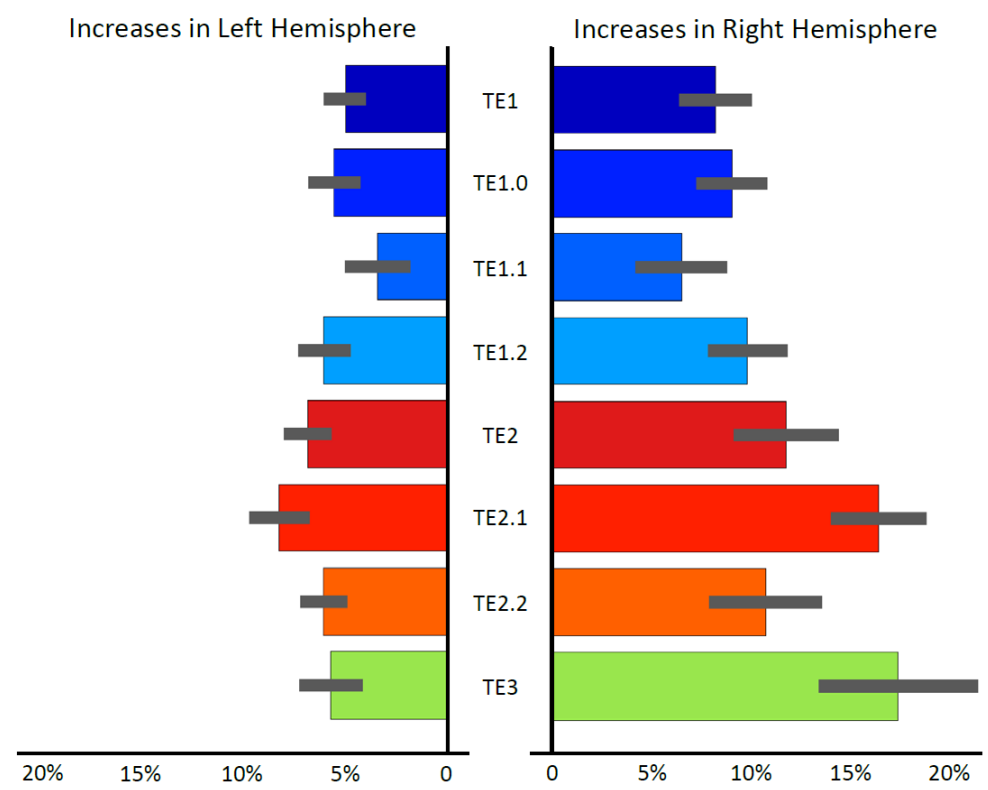

Figure 1. Changes in region-specific volumes between immediate and late postpartum. The colored bars show the mean increase in percent (\%) in blue hues for Te1 (consisting of Te1.0, Te1.1, and Te1.2), in red hues for $\mathrm{Te} 2$ (consisting of $\mathrm{Te} 2.1$ and $\mathrm{Te} 2.2$ ), and in green for Te3. The smaller gray bars indicate the standard error of the mean. 


\section{Discussion}

Contrasting the gray matter of the primary, secondary, and higher auditory cortex between immediate and late postpartum, we observed significant volume increases within all regions and subregions examined. In addition, there was a trend for a laterality effect within some regions, with larger postpartum increases within the right hemisphere compared to the left.

\subsection{Direction and Location of the Effect}

The direction of the effect (volume increases rather than decreases) after giving birth is in close agreement with the outcomes of most postpartum studies $[7,8,13,18-21]$; some of those studies $[7,13,19,21]$ were conducted using the same dataset as in the current study. With respect to the location of the effect, our findings corroborate prior reports of gray matter increases in auditory areas, as detected when applying voxel-based morphometry (VBM), both in independent and overlapping samples [7,8]. Nevertheless, our current study also extends those findings based on VBM, which is most sensitive to effects in the size and shape of the selected smoothing kernel (spatial smoothing is a common processing step in VBM analyses). In other words, VBM studies are less sensitive with respect to effects that are non-spherically shaped or smaller/larger than the smoothing kernel (which was $8 \mathrm{~mm}$ or $12 \mathrm{~mm}$ FWHM in the aforementioned studies). Thus, by specifically focusing on-and distinguishing between-regions and subregions of the auditory cortex, our study provides substantial and additional evidence for postpartum tissue changes within primary, secondary, and higher auditory areas. As such, it may also inform future studies investigating potential changes in auditory processing and sound perception during pregnancy and after giving birth [22].

\subsection{Possible Functional Links}

Overall, our findings might reflect adaptations of the mother's brain associated with listening for and interpreting her newborn's cries as well as other idiosyncratic sounds (gurgles, grunts, whines, squeals, etc.). The detected changes on the macro-anatomical level (i.e., gray matter increases) may reflect events on the micro-anatomical level (i.e., angiogenesis, dendritogenesis, gliogenesis, or synaptogenesis) which may drive and/or result from syncing a mother's brain to her newborn's means of communicating. The functional significance of the observed auditory changes in the maternal brain might thus be to foster a mother's ability to extract crucial information - not only in regards to physical and emotional states of her baby but also to degrees of urgency-in order to effectively intervene, altogether contributing to her newborn's wellbeing and flourishing. In addition to understanding what the different sounds of her baby signify (e.g., being hungry, sleepy, too hot, too cold, wet, in pain etc.), postpartum changes within the auditory cortex may also allow a new mother to recognize her own baby's cries [23], an ability reported to increase rapidly within a few days postpartum [24].

As discussed elsewhere, the maternal brain during the very early postpartum period constitutes an approximation of the pregnant brain $[7,19]$. Thus, apparent gray matter gain in auditory regions at late postpartum compared to immediate postpartum might also just reflect a restoration of brain tissue that may have been lost during pregnancy. Albeit research on this aspect is extremely sparse, one study [25] reported a significant gray matter decrease during pregnancy in various brain regions, including the left and right superior temporal gyrus, superior temporal sulcus, and middle temporal gyrus (i.e., regions that contain or are in close proximity to the auditory regions investigated in our study). In the same vein, our present findings within auditory regions might corroborate prior observations with respect to impaired or aberrant auditory functioning during pregnancy, that often resolve after giving birth, as reviewed in [22]. We have no record of auditory functioning whatsoever for our cohort, and we did not obtain a brain scan before pregnancy. However, it is certainly possible that tissue decreases within auditory regions took place 
during pregnancy - with or without noticeable impairment in auditory function-and simply reversed after giving birth and as such resulted in the detected tissue increases.

Finally, of course, it is also conceivable that these two possible mechanisms, brain reorganization and tissue restoration, are not mutually exclusive: The observed gray matter gain within the primary, secondary, and higher auditory cortex may be due to reorganization in some regions and due to restoration in others; and yet another set of regions might be affected by both mechanisms, either simultaneously or sequentially.

\subsection{Hemispheric Differences}

Our study revealed a trend for larger postpartum increases within some right-hemispheric regions compared to left-hemispheric regions. These structural laterality effects seem to be in agreement with functional laterality effects where infant crying (and also laughing) elicited stronger activations in auditory areas of the right hemisphere than the left hemisphere [3]. The auditory area where the left-right difference was most pronounced in our study was TE3, which corresponds to the posterior part of Brodmann Area 22. Since Brodmann Area 22 in the right hemisphere is closely involved in pitch, sound, and volume discrimination-while generating and understanding words are attributed to the left hemisphere (i.e., Wernicke's area) — the observed laterality trend might be related to the mother's ability to discern pitch, sound, and volume of her baby's crying. Future studies aiming to replicate this asymmetric growth effect in the very early postpartum stages may additionally consider investigating if the rightward growth becomes less pronounced (or even negated by a leftward growth) as the child transitions from crying to cooing to babbling to talking.

\section{Conclusions}

Our study provides evidence for postpartum tissue increases within primary, secondary, and higher auditory areas. The observed effects may reflect adaptations of the mother's brain in relation to interpreting her newborn's cries. However, given the small sample size and the relatively sparseness of studies in this field of research, follow-up research is clearly warranted to replicate (and potentially extent) the present findings as well as to evaluate their current interpretation.

Author Contributions: Conceptualization, E.L., F.K. and I.S.P.; methodology, E.L., F.K. and C.G.; formal analysis, F.K. and C.G.; data acquisition, M.G., J.E. and I.S.P.; writing-original draft, E.L.; writing-review and editing, E.L., F.K. and I.S.P.; visualization, E.L. and F.K.; project administration, E.L. and I.S.P.; funding acquisition, I.S.P. All authors have read and agreed to the published version of the manuscript.

Funding: This research was funded by the Swedish Research Council (K2014-54X-20642-07-4).

Institutional Review Board Statement: The study was conducted according to the guidelines of the Declaration of Helsinki and approved by the Institutional Review Board of Uppsala University (decision date: 2008-06-23; protocol number: 2008/156).

Informed Consent Statement: Informed consent was obtained from all subjects involved in the study.

Data Availability Statement: The conditions of our ethics approval do not permit public archiving of anonymized study data. Readers seeking access to the data should contact the corresponding author. Access may be granted to named individuals after completion of a formal data sharing agreement in accordance with ethical procedures governing the reuse of sensitive data.

Acknowledgments: The authors wish to thank all mothers participating in this study.

Conflicts of Interest: The authors declare no conflict of interest. 


\section{References}

1. Sander, K.; Frome, Y.; Scheich, H. FMRI activations of amygdala, cingulate cortex, and auditory cortex by infant laughing and crying. Hum. Brain Mapp. 2007, 28, 1007-1022. [CrossRef] [PubMed]

2. Young, K.; Parsons, C.; Elmholdt, E.-M.J.; Woolrich, M.W.; van Hartevelt, T.; Stevner, A.B.A.; Stein, A.; Kringelbach, M.L. Evidence for a Caregiving Instinct: Rapid Differentiation of Infant from Adult Vocalizations Using Magnetoencephalography. Cereb. Cortex 2015, 26, 1309-1321. [CrossRef]

3. Seifritz, E.; Esposito, F.; Neuhoff, J.; Lüthi, A.; Mustovic, H.; Dammann, G.; von Bardeleben, U.; Radue, E.W.; Cirillo, S.; Tedeschi, G.; et al. Differential sex-independent amygdala response to infant crying and laughing in parents versus nonparents. Biol. Psychiatry 2003, 54, 1367-1375. [CrossRef]

4. Bornstein, M.H.; Putnick, D.L.; Rigo, P.; Esposito, G.; Swain, J.; Suwalsky, J.T.D.; Su, X.; Du, X.; Zhang, K.; Cote, L.R.; et al. Neurobiology of culturally common maternal responses to infant cry. Proc. Natl. Acad. Sci. USA 2017, 114, E9465-E9473. [CrossRef] [PubMed]

5. Nelson, E.; Panksepp, J. Brain Substrates of Infant-Mother Attachment: Contributions of Opioids, Oxytocin, and Norepinephrine. Neurosci. Biobehav. Rev. 1998, 22, 437-452. [CrossRef]

6. Marlin, B.J.; Mitre, M.; D'Amour, J.A.; Chao, M.; Froemke, R.C. Oxytocin enables maternal behaviour by balancing cortical inhibition. Nat. Cell Biol. 2015, 520, 499-504. [CrossRef]

7. Luders, E.; Kurth, F.; Gingnell, M.; Engman, J.; Yong, E.-L.; Poromaa, I.S.; Gaser, C. From baby brain to mommy brain: Widespread gray matter gain after giving birth. Cortex 2020, 126, 334-342. [CrossRef]

8. Kim, P.; Leckman, J.F.; Mayes, L.C.; Feldman, R.; Wang, X.; Swain, J.E. The plasticity of human maternal brain: Longitudinal changes in brain anatomy during the early postpartum period. Behav. Neurosci. 2010, 124, 695-700. [CrossRef]

9. Morosan, P.; Rademacher, J.; Schleicher, A.; Amunts, K.; Schormann, T.; Zilles, K. Human Primary Auditory Cortex: Cytoarchitectonic Subdivisions and Mapping into a Spatial Reference System. NeuroImage 2001, 13, 684-701. [CrossRef]

10. Zachlod, D.; Rüttgers, B.; Bludau, S.; Mohlberg, H.; Langner, R.; Zilles, K.; Amunts, K. Four new cytoarchitectonic areas surrounding the primary and early auditory cortex in human brains. Cortex 2020, 128, 1-21. [CrossRef]

11. Morosan, P.; Schleicher, A.; Amunts, K.; Zilles, K. Multimodal architectonic mapping of human superior temporal gyrus. Z. Anat. Entwickl. 2005, 210, 401-406. [CrossRef]

12. Binder, J.R. The Wernicke area: Modern evidence and a reinterpretation. Neurology 2015, 85, 2170-2175. [CrossRef] [PubMed]

13. Luders, E.; Gaser, C.; Gingnell, M.; Engman, J.; Poromaa, I.S.; Kurth, F. Significant increases of the amygdala between immediate and late postpartum: Pronounced effects within the superficial subregion. J. Neurosci. Res. 2021, 99, 2261-2270. [CrossRef]

14. Kurth, F.; Jancke, L.; Luders, E. Integrating cytoarchitectonic tissue probabilities with MRI-based signal intensities to calculate volumes of interest. In Brain Morphometry: Methods and Clinical Applications; Spalletta, G., Gili, T., Piras, F., Eds.; Springer: New York, NY, USA; pp. 121-129.

15. Amunts, K.; Mohlberg, H.; Bludau, S.; Zilles, K. Julich-Brain: A 3D probabilistic atlas of the human brain's cytoarchitecture. Science 2020, 369, 988-992. [CrossRef] [PubMed]

16. Benjamini, Y.; Yekutieli, D. The control of the false discovery rate in multiple testing under dependency. Ann. Stat. 2001, 29, 1165-1188. [CrossRef]

17. Hochberg, Y.; Benjamini, Y. More powerful procedures for multiple significance testing. Stat. Med. 1990, 9, 811-818. [CrossRef] [PubMed]

18. Oatridge, A.; Holdcroft, A.; Saeed, N.; Hajnal, J.V.; Puri, B.K.; Fusi, L.; Bydder, G.M. Change in Brain Size during and after Pregnancy: Study in Healthy Women and Women with Preeclampsia. Am. J. Neuroradiol. 2002, 23, 19-26. [PubMed]

19. Luders, E.; Gingnell, M.; Poromaa, I.S.; Engman, J.; Kurth, F.; Gaser, C. Potential Brain Age Reversal after Pregnancy: Younger Brains at 4-6 Weeks Postpartum. Neuroscience 2018, 386, 309-314. [CrossRef]

20. Lisofsky, N.; Gallinat, J.; Lindenberger, U.; Kühn, S. Postpartal Neural Plasticity of the Maternal Brain: Early Renormalization of Pregnancy-Related Decreases? Neurosignals 2019, 27, 12-24. [CrossRef]

21. Luders, E.; Gaser, C.; Gingnell, M.; Engman, J.; Poromaa, I.S.; Kurth, F. Gray matter increases within subregions of the hippocampal complex after pregnancy. Brain Imaging Behav. 2021. [CrossRef]

22. Caras, M.L. Estrogenic modulation of auditory processing: A vertebrate comparison. Front. Neuroendocr. 2013, 34, 285-299. [CrossRef] [PubMed]

23. Formby, D. Maternal recognition of infant's cry. Dev. Med. Child Neurol. 1967, 9, 293-298. [CrossRef] [PubMed]

24. Bouchet, H.; Plat, A.; Levréro, F.; Reby, D.; Patural, H.; Mathevon, N. Baby cry recognition is independent of motherhood but improved by experience and exposure. Proc. R. Soc. B: Boil. Sci. 2020, 287, 20192499. [CrossRef] [PubMed]

25. Hoekzema, E.; Barba-Müller, E.; Pozzobon, C.; Picado, M.; Lucco, F.; García-García, D.; Soliva, J.C.; Tobena, A.; Desco, M.; Crone, E.; et al. Pregnancy leads to long-lasting changes in human brain structure. Nat. Neurosci. 2017, 20, 287-296. [CrossRef] 